\title{
Internetbasierte psychologische Behandlung bei Depressionen
}

\author{
Jan Philipp Klein ${ }^{a} \quad$ Thomas Berger ${ }^{b}$ \\ ${ }^{\text {a } Z e n t r u m ~ f u ̈ r ~ I n t e g r a t i v e ~ P s y c h i a t r i e ~ Z i P ~ g G m b H, ~ U n i v e r s i t a ̈ t ~ z u ~ L u ̈ b e c k, ~ D e u t s c h l a n d ~}$

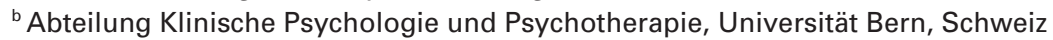

\section{Schlüsselwörter}

Internet - Computer - Depression - Psychotherapie . Internettherapie - Selbsthilfe - Geleitete Selbsthilfe . Prävention

\section{Zusammenfassung}

Depression ist die häufigste psychische Erkrankung, aber nur ein geringer Anteil der Betroffenen erhält eine angemessene Behandlung. Internetbasierte Interventionen stellen eine vielversprechende Ergänzung zu traditionellen Behandlungsformen dar, denn über das Internet können breite Bevölkerungsschichten mit wenig Aufwand erreicht werden. Die vorliegende Übersicht stellt den aktuellen Forschungsstand zu internetbasierten Interventionen bei Depressionen dar. Mehrere randomisierte kontrollierte Studien, Metaanalysen und Reviews legen nahe, dass mit strukturierten internetbasierten Interventionen, die regelmäßige Kontakte mit Therapeuten beinhalten (z.B. geleitete Selbsthilfeansätze, E-Mail- oder Chat-Therapien), Effekte erzielt werden können, die mit den Effekten von traditionellen Psychotherapien vergleichbar sind. Ungeleitete Selbsthilfeprogramme sind typischerweise mit hohen Abbrecherquoten und geringeren Effekten verbunden. Die zukünftige Forschung sollte sich vermehrt mit der Frage beschäftigen, wie internetbasierte Interventionen bei Depressionen optimal in die psychosoziale Versorgung integriert werden können. Mögliche Einsatzbereiche und die Einbettung internetbasierter Interventionen in das Versorgungssystem werden dargestellt und diskutiert.

\section{Keywords}

Internet - Computer - Depression - Psychotherapy . Internet-based treatment - Self-help - Guided self-help . Prevention

\section{Summary}

Internet-Based Psychological Treatments for Depression Depression is the most frequent mental health problem but only a small proportion of individuals in need receive adequate treatment. Internet-based depression treatment is a promising treatment alternative with which large populations can easily be reached. This review compiles the current evidence on internet-based treatments for depression. Several randomized controlled trials, meta-analyses, and reviews suggest that in structured internet-based treatments delivered with therapist support (e.g., guided self-help approaches, e-mail or chat therapies), effect sizes are comparable to those obtained in face-to-face psychotherapy. Internet-based interventions for depression without any support (selfguided programs) seem to result in considerably higher dropout rates and modest outcomes. Future research should evaluate optimal strategies for disseminating internet-based treatments for depression in routine health care settings. Health care areas to which internet-based treatments for depression can be applied are described and discussed.

\begin{tabular}{ll}
\hline KARGER & ( ) 2013 S. Karger GmbH, Freiburg \\
Fax +49 761 452 07 14 & Accessible online at: \\
Information@Karger.com & www.karger.com/ver \\
www.karger.com &
\end{tabular}




\section{Bedeutung internetbasierter psychologischer Interventionen bei Depressionen}

Im Jahr 2010 waren 6.9\% aller Europäer von einer Depression betroffen [Wittchen et al., 2011]. Depression ist die am häufigsten auftretende psychische Erkrankung, und die World Health Organization (WHO) sagt voraus, dass Depression im Jahr 2030 die häufigste Erkrankung überhaupt sein wird. Gleichzeitig erhalten weniger als $25 \%$ aller Patienten mit einer Depression eine angemessene Behandlung (29,2\% in Deutschland) [Wittchen et al., 2001]. In einem vielbeachteten Artikel [Kazdin und Blase, 2011] hat der ehemalige Präsident der American Psychological Association (APA) Alan E. Kazdin die Frage aufgeworfen, wie wir heute und in Zukunft die hohen Prävalenz- und Inzidenzraten psychischer Störungen reduzieren können. Kazdin zeigte auf, dass wir mit traditionellen Behandlungsformen wie der individuellen Psychotherapie nie in der Lage sein werden, dem enormen Bedarf nach psychosozialer Versorgung gerecht zu werden. Da psychisch belastete Menschen aus verschiedenen Gründen keine angemessene Behandlung erhielten (z.B. beschränkte Zahl an Therapeuten, beschränkter Zugang zu Therapeuten, zeitliche oder ökonomische Beschränkungen, Bequemlichkeit, Angst vor Stigmatisierung, hohe Hürde, einen Therapeuten aufzusuchen [Mohr et al., 2010]), bräuchten wir ein breites Portfolio an verschiedenen Versorgungs- und Interventionsmodellen, um möglichst viele Betroffene zu erreichen. $\mathrm{Zu}$ diesem Portfolio zählt Kazdin auch internetbasierte psychologische Interventionen. Diesen wurde in den letzten Jahren von verschiedenen Seiten viel Potenzial zugeschrieben [Hollon et al., 2002; Muñoz, 2010], insbesondere, weil sie leicht verbreitet und flexibel von zu Hause aus genutzt werden können, und weil Hemmungen und die hohe Hürde einen Therapeuten aufzusuchen, reduziert werden. Entsprechend hat sich das Feld internetbasierter psychologischer Interventionen in den letzten Jahren rapide entwickelt. In diesem Beitrag wollen wir einen Überblick über internetbasierte psychologische Interventionen bei Depressionen geben. Nach einer Einführung in die Begrifflichkeiten und die verschiedenen Ansätze wird die aktuelle Studienlage dargestellt. Schließlich werden Einsatzbereiche und die Einbettung internetbasierter Interventionen in die psychosoziale Versorgung diskutiert.

\section{Begriffe und Formen internetbasierter Interventionen}

Für internetbasierte psychologische Interventionen wurden in der Vergangenheit eine ganze Reihe unterschiedlicher Begriffe verwendet, wobei mit den verschiedenen Termini teils auch unterschiedliche Formen internetbasierter Interventionen gemeint sind. So werden mit Begriffen, die die Bezeichnung Therapie oder Psychotherapie enthalten wie «Internetpsychotherapie», «Online-Psychotherapie», «E-Mail-, Chat-Therapie» oder «Cybertherapie», oft Ansätze benannt,

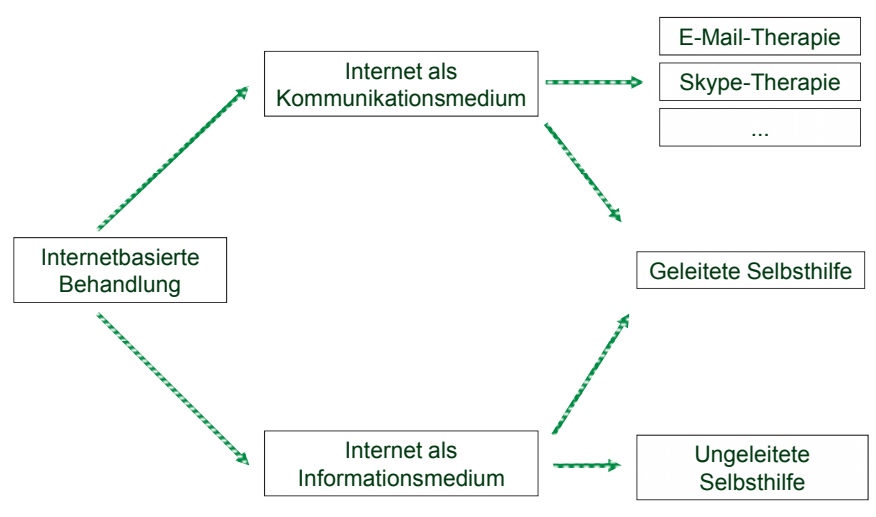

Abb. 1. Formen internetbasierter psychologischer Behandlung.

die das Internet ausschließlich oder vornehmlich als Kommunikationsmedium zwischen Therapeuten und Hilfesuchenden verwenden und letztlich Psychotherapie auf Distanz bedeuten.

Davon abzugrenzen ist die «internetbasierte Selbsthilfe», mit der meist nicht - wie der Begriff suggerieren könnte - der Austausch zwischen Betroffenen in Online-Selbsthilfegruppen bzw. -foren gemeint ist. Vielmehr erhalten Betroffene detaillierte, von Experten verfasste Instruktionen, wie sie Schritt für Schritt psychologische Interventionen selbständig umsetzen können. Im Gegensatz zur Internetpsychotherapie wird bei internetbasierten Selbsthilfeansätzen das Internet nicht als Kommunikations-, sondern als Informationsvermittlungsmedium verwendet.

Schließlich hat sich in den letzten Jahren der sogenannte geleitete Selbsthilfeansatz etabliert, in dem Patienten während der Arbeit mit einem webbasierten Selbsthilfeprogramm durch kurze wöchentliche Kontakte mit Therapeuten meist via E-Mail unterstützt werden. Dieser Ansatz kombiniert die Möglichkeiten des Internets als Informations- und Kommunikationsmedium, indem die meist standardisierte Vermittlung störungsspezifischer Methoden an ein Selbsthilfeprogramm delegiert wird und individuelle Probleme und Fragen mit Hilfe von Therapeuten bearbeitet werden [Berger und Andersson, 2009]. Diese Art der Intervention ist wiederum abzugrenzen von einer sogenannten gemischten Behandlung («blended treatment»), bei der traditionelle Behandlung im persönlichen Kontakt mit internetbasierten psychologischen Interventionen kombiniert wird. Traditionelle Psychotherapie im persönlichen Kontakt wird in diesem Zusammenhang auch als Face-to-Face (F2F)-Therapie bezeichnet. In Abbildung 1 sind die verschiedenen Formen internetbasierter psychologischer Interventionen zusammengefasst. Ein wesentlicher Unterschied zwischen den 3 dargestellten Formen besteht im Ausmaß des therapeutischen Kontakts, der in den verschiedenen Interventionen realisiert wird. Während bei ungeleiteten Selbsthilfeprogrammen Kliniker höchstens in einer diagnostischen Phase involviert sind, ist der zeitliche Aufwand der Therapeuten bei E-Mail- oder Chat-Therapien etwa gleich 
groß wie bei der F2F-Therapie [Vernmark et al., 2010]. Eine Zwischenstellung nehmen geleitete Selbsthilfeansätze ein, bei denen der zeitliche Aufwand der Therapeuten im Vergleich zu F2F-Therapien um etwa das 4- bis 5-Fache reduziert wird [Berger und Caspar, 2011].

\section{Vorteile und Herausforderungen}

Mit den verschiedenen Formen internetbasierter Interventionen sind jeweils Vor- und Nachteile verbunden. Ein wichtiger Vorteil von E-Mail-Therapien liegt darin, dass der Austausch mit dem Therapeuten bequem von zu Hause aus und $\mathrm{zu}$ frei wählbaren Zeiten stattfinden kann, und dass damit auch Patienten erreicht werden können, die aufgrund geographischer Gegebenheiten oder eingeschränkter Mobilität keinen Therapeuten finden. Internetpsychotherapie ist aber im Gegensatz zum Selbsthilfeansatz nicht skalierbar und braucht die gleichen therapeutischen Ressourcen wie traditionelle Psychotherapie. Ein Problem der Internetpsychotherapie ist auch, dass Psychotherapie, die ausschließlich über Kommunikationsmedien und Computernetzwerke erfolgt, zumindest in Deutschland nach der Musterberufsordnung für Ärzte verboten ist. Eine ähnliche Regelung findet sich auch in der Musterberufsordnung für psychologische Psychotherapeuten, die jedoch «begründete Ausnahmefälle unter Beachtung besonderer Sorgfaltspflichten» zulässt [Almer, 2008]. Eine Psychotherapie setzt also nach geltendem deutschen Recht im Regelfall einen persönlichen Kontakt voraus. Häufige Begründungen für diese Regelung sind, dass bei der Fernbehandlung wichtige diagnostische Informationen aus der nonverbalen Kommunikation fehlen, und dass bei Behandlungen auf Distanz nicht adäquat mit akuten Krisensituationen, insbesondere Suizidalität, umgegangen werden kann. In Modellprojekten und Studien, die gemäß der Musterberufsordnung erlaubt sind, werden deshalb suizidale Patienten vor Beginn der Behandlung ausgeschlossen, und meist wird auch ein individueller Notfallplan erarbeitet, in dem definiert ist, an wen sich $\mathrm{Pa}$ tienten vor Ort wenden können, wenn sie während der Behandlung in eine akute Krise geraten [Berger et al., 2011a]. Besonders relevant bei E-Mail- und Chat-Therapien sind auch datenschutztechnische Fragen, da Patienten in langen EMails sehr persönliche Informationen von sich preisgeben. Der Datenschutz ist selbstverständlich auch bei Selbsthilfeansätzen wichtig, allerdings sind die Eingabemöglichkeiten der Patienten in Selbsthilfeprogrammen oft beschränkt und die Informationen werden typischerweise anonymisiert und in einer gesicherten Umgebung gespeichert. Ein Vorteil von Selbsthilfeansätzen ist neben der Tatsache, dass große Populationen mit vergleichsweise geringen Kosten erreicht werden können, auch die konstante Qualität der Selbsthilfeprogramme. Allen Patienten können die gleichen standardisierten und empirisch validierten Inhalte geboten werden. Bei E-Mailoder Chat-Therapien sind Patienten sehr viel stärker von der
Qualität der Leistung der einzelnen Therapeuten abhängig. Diese Option hat jedoch den Vorteil, dass deutlich individueller auf die Probleme der Patienten eingegangen werden kann.

\section{Empirische Evidenz}

\section{Psychotherapie via Internet (z.B. E-Mail- oder Chat- Therapie)}

Während es zu gestützten und selbstangeleiteten internetbasierten Selbsthilfeinterventionen bei Depressionen zahlreiche Studien gibt, ist die Studienlage zu E-Mail- oder ChatTherapien sehr begrenzt. Einiges Aufsehen erregte vor allem eine in The Lancet veröffentlichte randomisierte kontrollierte Studie, in der 297 Patienten mit der Diagnose Depression in verschiedenen Hausarztpraxen in Großbritannien rekrutiert und in eine Chat-Therapie-Bedingung oder eine Kontrollgruppe randomisiert wurden [Kessler et al, 2009]. Die Chat-Therapie bestand aus 10 Sitzungen à 55 min, in denen ausgebildete Verhaltenstherapeuten stets online waren und über Textmeldungen in Echtzeit mit den Patienten kommunizierten. Die Kontrollgruppe erhielt von ihrem Hausarzt «treatment as usual». Nach Ende der Therapie waren 38\% der Probanden in der Chat-Therapie und 24\% der Probanden in der traditionellen Behandlung remittiert. Die Chancen auf eine Remission waren damit in der verhaltenstherapeutischen Chat-Therapie etwa doppelt so groß wie in der üblichen Hausarztbehandlung (Odds Ratio $=2,39$ ). Diese Unterschiede wurden auch 4 Monate nach Ende der Therapie festgestellt.

Im Gegensatz zur Chat-Therapie erfolgt die Kommunikation in der E-Mail-Therapie zeitversetzt bzw. asynchron; Patienten und Therapeuten sind also nicht gleichzeitig online und reagieren nicht sofort auf Nachrichten. E-Mail-Therapie wurde unseres Wissens bisher nur in einer kontrollierten Studie einer schwedischen Forschungsgruppe [Vernmark et al., 2010] untersucht. Dabei wurde die E-Mail-Therapie mit einer Warteliste und mit einem internetbasierten geleiteten Selbsthilfeansatz verglichen. In der E-Mail-Therapie-Bedingung wurden grundsätzlich dieselben kognitiv-verhaltenstherapeutischen Inhalte vermittelt wie im Selbsthilfeprogramm des geleiteten Ansatzes. Allerdings waren die Therapeuten freier in der Wahl und Ausgestaltung der verhaltenstherapeutischen Techniken und konnten so individueller auf die Bedürfnisse der Patienten eingehen. Probanden wurden über Medienberichte und Internetforen rekrutiert und nur eingeschlossen, wenn sie die Kriterien einer Major Depression erfüllten. In dieser Studie konnten statistisch signifikante Unterschiede der beiden Behandlungsbedingungen zur Wartelistenkontrollgruppe, aber kein signifikanter Unterschied zwischen der E-Mail-Therapie und dem geleiteten Selbsthilfeansatz gefunden werden. Die Zwischengruppeneffektstärke im Vergleich zur Wartelistenkontrollbedingung war bei der E-MailTherapie $(d=0,96)$ jedoch höher als bei der gestützten internetbasierten Behandlung $(\mathrm{d}=0,56)$. 
Insgesamt gibt es folglich noch wenig Studien zu Therapieformen, die das Internet nur als Kommunikationsmedium verwenden. Die beiden bestehenden Studien verweisen aber darauf, dass sich Patient und Therapeut nicht unbedingt im gleichen Raum befinden müssen, und dass Psychotherapie auch online und rein textbasiert funktionieren kann. In $\mathrm{Zu}$ kunft sollten neben E-Mail- oder Chat-Therapien auch Therapien untersucht werden, die über audiovisuelle internetbasierte Kommunikationsdienste (z.B. Skype) durchgeführt werden. In verschiedenen Studien zu Telefon-vermittelten Psychotherapien wurden bereits vielsprechende Ergebnisse auch für die Behandlung depressiver Patienten berichtet [Mohr et al., 2012]. Interessanterweise sind bei Telefon-vermittelten Therapien typischerweise die Abbrecherquoten geringer als bei F2F-Therapien, was darauf zurückgeführt wird, dass besonders Patienten, die gegenüber der Therapie ambivalente Gefühle hegen, bei der «bequemeren» Telefontherapie eher bereit sind, bei Schwierigkeiten weiterzumachen [Mohr et al., 2012].

\section{Internetbasierte ungeleitete Selbsthilfeprogramme}

Internetbasierte ungeleitete Selbsthilfeprogramme sind am ehesten mit bibliotherapeutischen Ansätzen vergleichbar, wobei die Ausgestaltungsmöglichkeiten größer sind als bei Selbsthilfebüchern. So können in Selbsthilfeprogrammen Audio- und Videobeiträge integriert werden, um auf diese Weise Fertigkeiten, beispielsweise der Achtsamkeit und der Entspannung zu vermitteln. Im Weiteren können die Inhalte der Programme an die individuellen Bedürfnisse und Probleme der Nutzer angepasst werden, beispielsweise indem auch Inhalte bezüglich vorhandener komorbider Probleme und Störungen zugeschaltet werden [Johansson et al., 2012]. Diese individuelle Anpassung kann auch erfolgen, indem auf individuelle Vorlieben der Nutzer für die Länge oder das Präsentationsformat der Inhalte im Rahmen eines simulierten Dialogs eingegangen wird [Meyer et al., 2009]. Eine neuere Entwicklung ist auch die Darbietung von Selbsthilfeinhalten in Smartphone-Apps [Ly et al., 2012] oder in Form sogenannter Serious Games, d.h. interaktiver Spielformate [Merry et al., 2012].

$\mathrm{Zu}$ internetbasierten ungeleiteten Selbsthilfeprogrammen bei depressiven Symptomen gibt es bereits eine ganze Reihe randomisierter kontrollierter Studien. Da mit Selbsthilfeprogrammen kostengünstig sehr breite Bevölkerungsgruppen erreicht werden können und ihr Einsatz vor allem auch im Bereich Prävention und Public Health erfolgt (siehe unten), weisen entsprechende Studien auch meist andere Merkmale auf als klassische Psychotherapiestudien. Dazu gehört, dass Depressionsdiagnosen oft nicht durch standardisierte Interviews gesichert, sondern Probanden eingeschlossen werden, die auf Selbsteinschätzungsfragebögen erhöhte Depressionswerte aufweisen. Während diese Vorgehensweise von Psychotherapieforschern teils kritisiert wird, verweisen Public-HealthForscher darauf, dass damit die externe Validität der Studie bzw. die Generalisierbarkeit der Ergebnisse auf einen wichti- gen Einsatzbereich von Selbsthilfeprogrammen erhöht wird. Denn aus der Public-Health-Perspektive sind niedrigschwellige Angebote wichtig, die von Menschen mit depressiven Symptomen (aber nicht zwingend mit einer Depressionsdiagnose) im Internet gefunden und sofort genutzt werden können.

Auffallend an den Studienergebnissen zu ungeleiteten Selbsthilfeprogrammen bei Depressionen ist zunächst die Heterogenität der gefundenen Effekte. Während in einigen Studien im Vergleich zu Wartelisten- und Placebokontrollgruppen mittlere bis große Effekte berichtet wurden [Berger et al., 2011a; Meyer et al., 2009], finden andere Studien kleine oder gar keine Effekte [Clarke et al., 2005; de Graaf et al., 2009]. Im Schnitt führte dies in einer Metaanalyse zum Ergebnis, dass ungeleitete Selbsthilfeprogramme einen kleinen aber statistisch signifikanten Effekt haben $(\mathrm{d}=0,28$; «numbers needed to treat» $=6,41$ ) [Cuijpers et al., 2011]. Diese Effektstärke gilt als niedrig, ist jedoch mit der Effektstärke zahlreicher gut etablierter medizinischer und psychopharmakologischer Behandlungen vergleichbar [Leucht et al., 2012].

Der wohl wichtigste Aspekt bei der Beurteilung der Wirksamkeit von ungeleiteten Selbsthilfeprogrammen ist, dass diese Behandlungsform typischerweise mit hohen Abbrecherund Drop-Out-Raten verbunden ist [Spek et al., 2007]. Die im Schnitt nur kleinen Effekte sind dabei ganz wesentlich durch die Tatsache bedingt, dass die Berechnungen auf der Basis von Intention-to-treat-Stichproben erfolgen und die vielen Abbrecher meist als «unverändert» in die Analysen einfließen. Für Completer-Stichproben werden in der Regel deutlich höhere Effekte berichtet [Titov, 2011].

Die Abbrecherquoten, die in den einzelnen Studien festgestellt wurden, sind auch ein wichtiger Anhaltspunkt, um die erwähnten differenziellen Effekte der Selbsthilfeprogramme zu erklären. Abbrecherquoten sind typischerweise dann besonders hoch, wenn die Zugangsschwelle zum Selbsthilfeprogramm sehr tief angesetzt wird. Ein Beispiel ist das australische Depressionsprogramm «MoodGym», das nach der Eingabe eines frei wählbaren Benutzernamens und Passworts unmittelbar und anonym genutzt werden kann. Die Zahl der Personen, die sich für solche «Open-Access»-Angebote registrieren, ist sehr hoch; gleichzeitig brechen aber auch viele die Intervention frühzeitig wieder ab [Christensen et al., 2004]. Im Gegensatz dazu wurden in Studien, die ein umfassenderes Abklärungsprozedere inklusive eines diagnostischen Interviews beinhalteten, deutlich geringere Abbrecherquoten und höhere Effekte festgestellt [Johansson und Andersson, 2012]. Mit dem beschriebenen Studientyp ist allerdings auch die Schwere der Symptombelastung konfundiert. Während Probanden in Studien zu Open-Access-Programmen typischerweise nur leicht belastet waren, wurden in Studien, die ein diagnostisches Interview beinhalten, teils nur Patienten eingeschlossen, die die Kriterien einer Depression erfüllen. Bei den stärker belasteten Patienten im zweiten Studientyp besteht einerseits mehr Raum für eine positive Veränderung, was die höheren Effekte erklären könnte, andererseits kann der stär- 
kere Leidensdruck auch dazu führen, dass Patienten weniger abbrechen. Ein weiterer Grund für die heterogenen Ergebnisse bei ungeleiteten Selbsthilfeprogrammen könnten die unterschiedlichen Inhalte und die Länge der verschiedenen Selbsthilfeprogramme sein. So wurden für das sehr umfassende kognitiv-verhaltenstherapeutische Selbsthilfeprogramm deprexis $^{\circledR}$, das im deutschsprachigen Raum entwickelt und getestet wurde, höhere Effekte gefunden als für kürzere Selbsthilfeprogramme, die z.B. nur psychoedukative Elemente beinhalten [Meyer et al., 2009].

Zusammenfassend kann festgehalten werden, dass ungeleitete internetbasierte Selbsthilfeprogramme bei Depressionen im Schnitt zwar signifikante aber nur kleine Effekte haben und typischerweise mit hohen Abbrecherquoten verbunden sind. Die Abbrecherquoten und Effekte scheinen davon abzuhängen, wie Patienten Zugang zu den Selbsthilfeprogrammen gewährt wird. Die Compliance der Patienten und die Effekte sind dann besonders günstig, wenn der Zugang zum Programm erst nach einer umfassenden diagnostischen Abklärung erfolgt.

\section{Internetbasierte geleitete Selbsthilfeprogramme}

Das Ergebnis der oben genannten Metaanalyse zu selbstangeleiteten Behandlungen entspricht im Wesentlichen dem Ergebnis einer weiteren Metaanalyse aus dem Jahr 2009 [Andersson und Cuijpers, 2009]. Ein besonderer Fokus dieser Studie war der Vergleich von selbstangeleiteten mit gestützten internetbasierten Behandlungen. Auch für die 15 in dieser Metaanalyse zusammengefassten Studien wurde meist aus der allgemeinen Bevölkerung und über Krankenkassen rekrutiert. Nur eine Studie schloss Patienten ein, die über Hausärzte rekrutiert wurden. Selbstangeleitete Behandlungen zeigten in dieser Studie eine deutlich geringere Effektstärke ( $d=$ $0,25)$ als geleitete Behandlungen $(d=0,61)$.

Die Unterschiede in den Effektstärken der ungeleiteten und geleiteten Selbsthilfeprogramme sind im Wesentlichen auf deutlich geringere Abbrecherquoten in geleiteten Behandlungen zurückzuführen [Spek et al., 2007]. Im Gegensatz zu ungeleiteten Selbsthilfeprogrammen liegt die Drop-outRate in geleiteten Selbsthilfeprogrammen im Schnitt bei nur ca. $10 \%$. Das entspricht etwa Abbrecherquoten, die auch in F2F-Psychotherapien berichtet werden. Die kurzen, aber regelmäßigen, meist wöchentlichen therapeutischen Nachrichten in geleiteten Behandlungen sind vor allem darauf ausgerichtet, Patienten Struktur zu geben und sie zu motivieren, mit dem Selbsthilfeprogramm zu arbeiten. Entsprechende Ansätze wurden auch schon vor dem Internetzeitalter z.B. mit Selbsthilfebüchern und kurzen telefonischen Kontakten mit Therapeuten realisiert. Für diese ursprüngliche Form der geleiteten Selbsthilfe («guided self-help») liegen bereits viele Studien vor. Besonders interessant ist in diesem Zusammenhang eine Metaanalyse, in die nur Studien eingeschlossen wurden, in denen der geleitete Selbsthilfeansatz bei Depressionen und Angststörungen direkt mit F2F-Therapien vergli- chen wurde. In den meisten dieser Studien wurden traditionelle und nicht internetbasierte Formen geleiteter Selbsthilfebehandlungen untersucht [Cuijpers et al., 2010]. Die über die verschiedenen Studien hinweg gemittelte Zwischengruppeneffektstärke lag bei $d=-0,03$, was bedeutet, dass F2F-Therapien und geleitete Selbsthilfeansätze gleich wirksam sind. Die gute Wirkung der internetbasierten Ansätze ist also wahrscheinlich weniger auf das Medium Internet zurückzuführen als auf die Tatsache, dass geleitete Selbsthilfe grundsätzlich eine wirksame Therapieform bei Angststörungen und Depressionen ist.

Internetbasierte geleitete Selbsthilfeansätze unterscheiden sich bezüglich der Intensität des therapeutischen Kontakts. Es gibt die sogenannten Low-intensity-guidance-Ansätze, in denen die Therapeuten insgesamt etwa $2 \mathrm{~h}$ investieren und den Patienten vor allem Struktur geben und sie motivieren [Titov, 2011]. Eine typische Nachricht eines Therapeuten in diesem Ansatz beinhaltet Verstärkung (z.B. «Ich habe mich sehr gefreut zu sehen, dass Sie die ersten beiden Sitzungen des Selbsthilfeprogramms durchgearbeitet und auch die Hausaufgaben gemacht haben. Weiter so!») und kurze Hinweise auf das weitere Vorgehen (z.B. «Gerne können Sie nun mit der dritten Sitzung beginnen, in welcher es um negative Gedankenmuster geht»). Daneben gibt es High-intensityguidance-Ansätze, in denen Therapeuten mit den Patienten intensiver im Austausch stehen und wie in E-Mail-Therapien stärker auch inhaltlich therapeutisch arbeiten. $\mathrm{Zu}$ diesen intensiver betreuten Interventionen gehört der sogenannte Interapy-Ansatz, der ursprünglich zur Behandlung der posttraumatischen Belastungsstörung (PTBS) entwickelt wurde, inzwischen aber bei verschiedenen Störungen, auch der Depression, eingesetzt und erforscht wird [Lange et al., 2003; Knaevelsrud und Maercker, 2007; Ruwaard et al., 2009, 2012; Preschl et al., 2012]. Dieser Ansatz beinhaltet insbesondere verschiedene strukturierte Schreib- und Hausaufgaben. Patienten werden z.B. in der ersten Behandlungsphase gebeten, innerhalb von ca. 45 min einen Essay darüber zu schreiben, wann sie in der Vergangenheit depressive Symptome erlebt haben, und ob es Ursachen oder auslösende Ereignisse für die negativen Gefühle und Gedanken gab [Wagner und Lange, 2008]. Der im Vergleich zu den wenig intensiv betreuten Interventionen höhere Aufwand der Therapeuten hat mehrere Gründe: Zum einen erhalten die Patienten auf die Schreibund Hausaufgaben von den Therapeuten individuell und detailliert Rückmeldung. Zum anderen wird der Fokus grundsätzlich stärker auf das gemeinsame Erarbeiten der Inhalte der Intervention gelegt. So überlegen die Patienten z.B. gemeinsam mit den Therapeuten, wie bestimmte negative Gedanken in der Realität bzw. mit einem Verhaltensexperiment überprüft werden können [Wagner und Lange, 2008]. Für diesen intensiver geleiteten Ansatz liegen bereits Studien zur Behandlung der Depression vor, in denen eine deutliche Reduktion der Depressionssymptomatik mit großen Effektstärken im Vergleich zu Kontrollgruppen festgestellt wurde [Lange et 
al., 2005; Ruward et al., 2012]. Die berichteten großen Effektstärken im Vergleich zu Wartelisten sind dabei tendenziell leicht höher als bei Low-intensity-guidance-Ansätzen. Entsprechende Vergleiche sind aber mit Vorsicht zu betrachten, da es bisher keine direkten Vergleiche zwischen Low- und High-intensity-guidance-Ansätzen gibt. Mit dem InterapyAnsatz wurde auch die unseres Wissens erste Studie realisiert [Preschl et al., 2012], in der ein internetbasierter Ansatz bei Depressionen direkt mit F2F-Psychotherapie verglichen wurde. Erste Ergebnisse dieser Studie, in der Teilnehmer aus der Allgemeinbevölkerung über Zeitungsannoncen und Internetseiten rekrutiert wurden, zeigen, dass die beiden Ansätze gleich wirksam sind.

Insgesamt kann festgehalten werden, dass sich internetbasierte geleitete Selbsthilfeansätze bisher als ausgesprochen wirksam erwiesen haben. Effektstärken und Abbrecherquoten liegen im Bereich der Ergebnisse, die auch in Studien zu F2F-Therapien berichtet werden. Zu berücksichtigen ist allerdings, dass in den meisten der erwähnten Untersuchungen die Teilnehmer aus der Allgemeinbevölkerung über Annoncen, Zeitungsberichte oder Internetseiten rekrutiert wurden. Untersucht wurden also selbstselegierte Stichproben mit Probanden, die für den internetbasierten Ansatz möglicherweise besonders geeignet und motiviert waren. Die Frage, ob internetbasierte geleitete Selbsthilfeprogramme bei Depressionen auch in der Praxis, d.h. in der routinemäßigen Anwendung funktionieren, wurde noch wenig untersucht. Erste sogenannte Effectiveness-Studien verweisen aber darauf, dass internetbasierte Ansätze bei Depressionen und Angststörungen auch in der Routinepraxis erfolgversprechend sind [Ruward et al., 2012; Hedman et al., 2011, 2013].

\section{Die Rolle der Therapeuten und der therapeutischen Beziehung}

Aus den darstellten Ergebnissen zu den verschiedenen internetbasierten Interventionen geht hervor, dass den Therapeuten auch in internetbasierten Ansätzen eine wichtige Rolle zukommt. Die Überlegenheit von geleiteten Selbsthilfebehandlungen gegenüber ungeleiteten Programmen bezüglich Wirksamkeit und Abbrecherquoten wurde in Metaanalysen gezeigt [Spek et al., 2007; Andersson und Cuijpers, 2009]. Dabei bestehen auch gewisse Hinweise, dass die therapeutische Unterstützung bei Depressionen im Vergleich zu anderen Störungen besonders wichtig ist. Während für internetbasierte Programme bei Angststörungen teilweise überhaupt keine Wirkungsunterschiede zwischen ungeleiteten und geleiteten Selbsthilfeprogrammen festgestellt wurden [Berger et al., 2011b], treten Unterschiede bei der Behandlung von Depressionen deutlicher und konsistenter hervor [Berger et al., 2011a; Johansson und Andersson, 2012].

Unklar ist derzeit, welches Ausmaß an ergänzenden Kontakten ideal ist. Aufgrund der bestehenden Erkenntnisse ist bekannt, dass kurze minimale Kontakte bezüglich Compliance und Wirkung eine relativ große Wirkung haben; jeder zusätzliche Kontakt hat jedoch nur einen geringen zusätzlichen Nutzen (abnehmender Grenznutzen des therapeutischen Kontakts). Einen nicht zu unterschätzenden Effekt scheint dabei auch schon der therapeutische Kontakt während der diagnostischen Phase zu haben. In einem systematischen Review zu internetbasierten Ansätzen bei Depressionen teilten Johansson und Andersson [2012] die Originalstudien in 4 Typen ein. Gar keinen Kontakt hatten Probanden in Studien zu ungeleiteten Selbsthilfeprogrammen ohne diagnostisches Interview. Die gemittelte Effektstärke lag bei diesem Studientyp bei Cohens $d=0,21$. Beinhalteten die ungeleiteten Selbsthilfeinterventionen einen therapeutischen Kontakt in der Diagnostikphase vor Beginn der Behandlung, lag die Effektstärke bei $\mathrm{d}=0,44$. Für geleitete Selbsthilfeinterventionen wurde eine gemittelte Effektstärke von $d=0,58$ festgestellt, wenn kein diagnostisches Interview stattfand, und eine Effektstärke von $\mathrm{d}=0,76$, wenn sowohl vor Beginn als auch während der Behandlung therapeutische Kontakte realisiert wurden. Neben der therapeutischen Begleitung während der Behandlung können also auch schon Kontakte vor der Behandlung einen positiven Effekt haben.

Eine Frage, die im Zusammenhang mit internetbasierten Interventionen oft gestellt wird ist, ob auch in internetbasierten Ansätzen eine gute Therapiebeziehung aufgebaut werden kann, und ob ihr eine ähnliche Bedeutung zukommt wie in F2F-Psychotherapien. Empirisch relativ gut gesichert ist dabei die Erkenntnis, dass in internetbasierten Ansätzen gemäß Patienteneinschätzung in der Regel eine gute therapeutische Beziehung etabliert werden kann. Werden die in internetbasierten Interventionen gewonnenen Mittelwerte auf Fragebögen, die typischerweise zur Messung der Therapiebeziehung eingesetzt werden (z.B. «Working Alliance Inventory»), mit den in F2F-Therapien berichteten Mittelwerten verglichen, sind sie praktisch identisch. Dies zeigte sich beispielsweise in der bereits erwähnten Studie [Preschl et al., 2012] zum Vergleich der Interapy mit einer F2F-Psychotherapie bei Depressionen. Hier wurden mit dem Working Alliance Inventory in beiden Bedingungen hohe Allianzwerte und keine Unterschiede zwischen den Gruppen festgestellt. Gemischter ist die Studienlage bezüglich der Frage, ob die Therapiebeziehung auch mit dem Therapieergebnis zusammenhängt. In der erwähnten Depressionsstudie wurden nur geringe und nicht signifikante Zusammenhänge gefunden, während in einer anderen Interapy-Studie zur Behandlung der PTBS zumindest die später im Therapieprozess erfasste Therapiebeziehung statistisch signifikant mit dem Behandlungserfolg korrelierte [Knaevelsrud und Maercker, 2007].

Wenig erforscht ist die Frage, was eine Therapiebeziehung in Online-Behandlungen genau ausmacht und inwiefern sie sich von der Therapiebeziehung in einer traditionellen Therapie unterscheidet. In eigenen Studien zeigte sich, dass Patienten in Online-Behandlungen die Therapiebeziehung nahezu 
nie schlecht einschätzen - die Therapiebeziehung war mit allen Patienten gut. Dies spricht einerseits für die aus der allgemeinen Forschung zu Online-Beziehungen stammende These, dass das Gegenüber in Online-Beziehungen aufgrund eingeschränkter Informationen (z.B. aufgrund fehlender nonverbaler Informationen) idealisiert wird bzw. im Vergleich zu F2F-Therapien stärker entsprechend den eigenen Vorstellungen und Wünschen geformt werden kann [Walther, 1996]. Andererseits könnte die eingeschränkte Varianz auch die relativ geringen Zusammenhänge zwischen der Therapiebeziehung und dem Therapieergebnis in Online-Interventionen erklären. Die im Vergleich zu traditionellen Therapien eingeschränkten Gestaltungsmöglichkeiten der Therapiebeziehung können sowohl ein Nachteil wie auch ein Vorteil sein, denn die Therapiebeziehung kann in F2F-Therapien auch zu vielen Problemen bis hin zu Therapieabbruch und Missbrauch führen [Caspar, 2004]. Entsprechende Gefahren werden in Online-Therapien reduziert.

\section{Inhalte der internetbasierten Interventionen}

Die meisten internetbasierten Interventionen bei Depressionen basieren auf empirisch-validierten, kognitiv-verhaltenstherapeutischen Manualen. Aufgrund der starken Strukturierung, Standardisierung und Direktivität eignet sich dieser Ansatz besonders für die Vermittlung via Internet. Wie die traditionelle Verhaltenstherapie enthalten die internetbasierten Interventionen viele psychoedukative Elemente, was den aktuellen Leitlinien zur Behandlung von Depression entspricht [DGPPN et al., 2009]. Inzwischen wurde aber auch eine internetbasierte geleitete Selbsthilfeintervention evaluiert, die auf einem psychodynamischen Ansatz zur Behandlung der Depression basiert [Johansson et al., 2012]. Auch für diese Intervention wurden im Vergleich zu einer Kontrollgruppe große Effektstärken festgestellt. Diese Ergebnisse zeigen, dass auch andere als verhaltenstherapeutische Ansätze erfolgreich via Internet realisiert werden können. Ein weiteres Beispiel dafür ist das bereits erwähnte in Deutschland entwickelte Programm deprexis, das einen integrativen Ansatz verfolgt [Meyer et al., 2009]. Neben kognitiv-verhaltenstherapeutischen Elementen beinhaltet das Programm z.B. auch emotionsfokussierte Interventionen und die Arbeit mit Träumen. In zukünftigen Studien sollten auch die spezifischen Effekte der einzelnen Behandlungskomponenten untersucht werden.

Die meisten der evaluierten Programme sind störungsspezifisch und auf die Veränderung der depressiven Symptomatik ausgerichtet. In jüngerer Zeit wurden aber auch vermehrt Programme evaluiert, die störungsübergreifend sind und auch auf die Veränderung komorbider Probleme und Störungen abzielen. Dazu gehört ein von einer australischen Forschungsgruppe entwickelter internetbasierter transdiagnostischer Ansatz bei Depressionen und Angststörungen [Titov et al., 2011]. Diese geleitete Selbsthilfeintervention hat sich sowohl bei
Depressionen als auch bei Angststörungen als wirksam erwiesen. Ein weiterer Ansatz, der von der bereits erwähnten schwedischen Forschungsgruppe verfolgt wurde, umfasst eine individualisierte Intervention («tailored treatment»). Das Selbsthilfeprogramm wird aus einem Pool verschiedener Interventionsmodule zusammengestellt und auf die in einem diagnostischen Interview erfassten Probleme und Störungen des Patienten abgestimmt [Carlbring et al., 2011]. Ein Patient, der gleichzeitig unter einer Depression und generalisierten Angststörungen leidet, erhält neben Modulen, die auf die Veränderung der Depression abzielen, auch Interventionsmodule, die für die Therapie der generalisierten Angststörung entwickelt wurden (z.B. Sorgenkonfrontation, Grübelstuhl). In einer Studie von Johanson et al. [2012] wurde festgestellt, dass dieser individualisierte Ansatz bei schwer depressiven Patienten der standardisiert-störungsspezifischen Intervention überlegen ist. Das ist die bisher einzige Studie, in der bei einem Vergleich zwischen 2 internetbasierten Ansätzen Unterschiede festgestellt wurden.

\section{Einsatzbereiche und Einbettung in Phasen der psychosozialen Versorgung}

Internetbasierte Interventionen können in unterschiedlichen Phasen der psychosozialen Versorgung eingesetzt werden. Tabelle 1 gibt einen Überblick. Im Folgenden werden mögliche Einsatzbereiche und die Einbettung internetbasierter Interventionen in die psychosoziale Versorgung diskutiert.

Prävention und frühe Intervention: Präventionsangebote zur Vermeidung psychischer Erkrankungen richten sich in der Regel an (noch) gesunde Personen oder Risikogruppen, die zumindest die Kriterien einer psychischen Störung (noch) nicht erfüllen. Entsprechend gering ist der Leidensdruck und umso wichtiger ist es, den Zugang zu präventiven Interventionen möglichst einfach und attraktiv zu gestalten. Internetbasierte Interventionen sind ein interessantes Instrument zur Prävention von depressiven Erkrankungen, weil sie eine niedrige Zugangsschwelle aufweisen und nach der recht aufwendigen Entwicklung entsprechender Programme vergleichsweise einfach auch einer großen Population zur Verfügung gestellt werden können (hohe Skalierbarkeit). Die meisten der oben erwähnten Studien zu internetbasierten Selbsthilfeprogrammen weisen auch Merkmale auf, die typisch für präventive Maßnahmen sind. Zu diesen Merkmalen gehört, dass die Intervention der Eindämmung bereits vorhandener, aber relativ leicht ausgeprägter depressiver Symptome dienen, und dass sie bevölkerungswirksam sein soll, d.h. in der Grundgesamtheit von Menschen mit depressionsbezogenen Risikofaktoren zu messbaren Erfolgen führt. Selbsthilfeinterventionen, für die Patienten mit erhöhten Depressionssymptomen aus der Bevölkerung rekrutiert werden, erfüllen diese Kriterien. Entsprechend kann davon ausgegangen werden, dass die oben berichteten kleinen Effektstärken, die für Programme ohne 
Tab. 1. Überblick der Einsatzmöglichkeiten internetbasierter Interventionen in unterschiedlichen Phasen der psychosozialen Versorgung

\begin{tabular}{|c|c|}
\hline Phasen der Versorgung & Beispiele für Interventionen \\
\hline Prävention & $\begin{array}{l}\text { niedrigschwelliger Zugang zu ungeleiteter Selbsthilfe } \\
\text { Vermittlung von Selbsthilfestrategien via E-Mail }\end{array}$ \\
\hline \multicolumn{2}{|l|}{ Therapie } \\
\hline $\begin{array}{l}\text { Frühintervention im Rahmen einer gestuften } \\
\text { Versorgung }\end{array}$ & $\begin{array}{l}\text { ungeleitete Selbsthilfe als erster Behandlungsschritt, geleitete } \\
\text { Selbsthilfe und traditionelle Psychotherapie bei ausbleibendem } \\
\text { Behandlungserfolg }\end{array}$ \\
\hline Einsatz in der Primärversorgung & $\begin{array}{l}\text { Unterstützung von Selbsthilfeprogramm durch motivierende } \\
\text { Gespräche mit dem Hausarzt }\end{array}$ \\
\hline $\begin{array}{l}\text { Vorbereitung einer Psychotherapie während } \\
\text { der Wartezeit }\end{array}$ & $\begin{array}{l}\text { Vermittlung von Inhalten und Übungen mittels ungeleiteter Selbst- } \\
\text { hilfe, diese werden später auch Gegenstand der Psychotherapie }\end{array}$ \\
\hline $\begin{array}{l}\text { Ergänzung einer Psychotherapie oder } \\
\text { Psychopharmakotherapie (blended treatment) }\end{array}$ & $\begin{array}{l}\text { Unterstützung der traditionellen Behandlung, z.B. durch E-Mails, } \\
\text { ungeleitete oder geleitete Selbsthilfe }\end{array}$ \\
\hline Behandlung von unterversorgten Gruppen & $\begin{array}{l}\text { kultursensible muttersprachliche Behandlung bei Migranten } \\
\text { Behandlung im Haus der Patienten, z.B. bei älteren Menschen } \\
\text { oder Gehbehinderten }\end{array}$ \\
\hline Behandlung in medizinischen Settings & $\begin{array}{l}\text { Prävention und Behandlung von komorbiden Depressionen, } \\
\text { z.B. bei Diabetes oder Herzinfarkt }\end{array}$ \\
\hline $\begin{array}{l}\text { Rückfallprophylaxe nach Abschluss einer } \\
\text { Behandlung }\end{array}$ & Chat- oder E-Mail-Brücke nach Abschluss der Behandlung \\
\hline
\end{tabular}

Therapeutenkontakt ermittelt wurden, eine gute Schätzung für die Wirkung internetbasierter präventiver Interventionen darstellen.

In einer Studie, die explizit auf die Prävention depressiver Erkrankungen bei Erwachsenen ausgerichtet war [Morgan et al., 2012], bot eine australische Arbeitsgruppe Teilnehmern mit bestehender depressiver Symptomatik randomisiert eine 6-wöchige Behandlung an. Eine depressive Störung war bei den Teilnehmern ausgeschlossen worden. Alle Teilnehmer erhielten 2-mal wöchentlich E-Mails. In der Interventionsgruppe wurden in den E-Mails Selbsthilfestrategien vermittelt. In der Kontrollgruppe wurden lediglich Informationen über Depressionen vermittelt. Im Ergebnis konnte ein geringer Effekt auf das Depressionsrisiko («number needed to treat» (NNT) 25) und die depressive Symptomatik (gemessen im «patient health questionnaire» $(\mathrm{PHQ}): \mathrm{d}=0,17$ ) gezeigt werden.

Beforscht werden internetbasierte Interventionen auch in der Prävention von depressiven Erkrankungen bei Jugendlichen. Dabei wird dieser Therapieform wegen der hohen Internetaffinität der Jugendlichen und jungen Erwachsenen eine besondere Bedeutung zugeschrieben. In einem Review aus dem Jahr 2010 [Calear und Christensen, 2010] kamen die Autoren zum dem Schluss, dass die vorliegenden Studien zu Präventionsinterventionen bei Jugendlichen von unterschiedlicher Qualität sind, jedoch erste Hinweise auf deren Wirksamkeit bieten. Bei diesem Review wurden neben internetbasierten Interventionen auch Angebote an Schulen, bei Hausärzten oder in psychiatrischen Ambulanzen berücksichtigt. Eine norwegische Arbeitsgruppe untersuchte in einer kürzlich erschienenen Studie mit jungen Erwachsenen [Lintvedt et al., 2013] ein ungeleitetes Selbsthilfeprogramm. Die Therapie beinhaltete eine Kombination aus der norwegischen Übersetzung der bereits beschriebenen australischen Intervention
MoodGym und einem Psychoedukationsprogramm. Für diese Intervention konnte im Vergleich zu einer Wartelistenbedingung eine Effektstärke von $\mathrm{d}=0,57$ gezeigt werden .

\section{Therapie}

Einsatz in der Primärversorgung: Der Einsatz internetbasierter Interventionen in der Primärversorgung ist aus verschiedenen Gründen attraktiv. Erstens sind immer weniger Ärzte bereit, leicht bis mittelschwer depressive Patienten medikamentös zu behandeln, auch weil Metaanalysen nahelegen, dass Antidepressiva bei leichten und mittelschweren Depressionen nicht besser wirken als Placebo [Fournier et al., 2010; Kirsch et al., 2008]. Zweitens ist gut belegt, dass Patienten in westlichen Ländern psychologische gegenüber medikamentösen Behandlungen präferieren [Kessing et al., 2005; Raue et al., 2009]. Die Bedeutung der selbstangeleiteten Behandlung wird auch in der «Nationalen Versorgungsleitlinie Depression» hervorgehoben [DGPPN et al, 2009]. Drittens haben sich internetbasierte Interventionen, wie oben dargestellt, besonders dann als wirksam erwiesen, wenn sie nach einer diagnostischen Abklärung verschrieben wurden. Ein Modell, in dem Hausärzte nach einer diagnostischen Abklärung internetbasierte psychologische Interventionen verschreiben, erscheint also vielversprechend. Diese Annahme wird auch durch 2 bereits erwähnte Studien unterstützt [Kessler et al., 2009; Ruward et al., 2012].

Im Jugendlichen-Bereich gibt es eine weitere niederländische Studie [van Voorhees et al., 2009], in der die Wirkung einer motivierenden Gesprächsführung durch den Hausarzt auf die Effektivität einer onlinebasierten Intervention zur Depressionsbehandlung untersucht wurde. Dabei wurde die motivierende Gesprächsführung durch Hausärzte mit einer kurzen Beratung durch Hausärzte verglichen. Alle Patienten 
erhielten eine internetbasierte Intervention. Eine Überlegenheit der motivierenden Gesprächsführung konnte jedoch am Ende der Intervention nicht gezeigt werden. Die Überlegenheit zeigte sich erst zum 6-monatigen Nachbeobachtungszeitpunkt.

Einsatz in psychiatrisch-psychotherapeutischen Settings: Auch in psychiatrisch-psychotherapeutischen Settings können internetbasierte Interventionen das traditionelle Angebot ergänzen und erweitern. Dabei kann zwischen therapievorbereitenden und therapiebegleitenden Interventionen unterschieden werden. Vorbereitend ist eine Intervention, wenn während der Wartezeit auf eine Psychotherapie Inhalte vermittelt werden, die in ähnlicher Form auch im Lauf der Behandlung thematisiert werden. Dann ist die internetbasierte Intervention auch mehr als «nur» eine Überbrückung der Wartezeit. Bestenfalls kann die anschließende Psychotherapie sogar schneller zu einem Erfolg kommen, weil der Patient auf bestimmte Ideen schon vorbereitet ist und mit einzelnen Techniken schon eigene Erfahrung gesammelt hat. Einen wissenschaftlichen Beleg für diese Annahme gibt es jedoch leider nicht. Interessant in diesem Zusammenhang ist das Ergebnis einer Studie, die die Auswirkungen einer internetbasierten Intervention auf die Einstellung zur Psychotherapie untersuchte. Dabei zeigte die internetbasierte Intervention keine Auswirkungen auf die Einstellung zur Psychotherapie [Moritz et al., 2013]. Im Weiteren können internetbasierte Selbsthilfeprogramme im Sinne eines blended treatment den Patienten auch therapiebegleitend als zusätzliches Element während einer ambulanten, stationären oder tagesklinischen Behandlung angeboten werden. Die Einbettung internetbasierter Interventionen in traditionelle psychotherapeutisch-psychiatrische Behandlungen erscheint auch deshalb sinnvoll, weil in diesem Setting die Patientensicherheit optimal gewährleistet werden kann. Viele Therapeuten berichten auch, dass sie beispielsweise im Rahmen einer traditionellen Behandlung EMails als Brücken zwischen Therapieterminen einsetzen. Umso erstaunlicher ist, dass in diesem Bereich noch keine veröffentlichten Studien existieren.

Einsatz bei unterversorgten Patientengruppen: Ein weiteres wichtiges Einsatzgebiet für internetbasierte Interventionen sind Patientengruppen, die gegenwärtig nicht ausreichend versorgt sind. Dazu zählen beispielsweise ältere Menschen und Migranten. Ältere Menschen sind häufig weniger mobil und bevorzugen daher eine Behandlung in ihrem Wohnumfeld. Bei der Psychotherapie von Migranten müssen nicht nur die Sprachbarriere sondern auch kulturelle Unterschiede beachtet werden. Besonders zu computer- und internetgestützten Behandlungen bei älteren Menschen gibt es bereits einiges an Forschungsmaterial [Preschl et al., 2011].

Einsatz in anderen medizinischen Settings: Internetbasierte Interventionen bei Depressionen können in verschiedenen anderen medizinischen Settings nützlich sein. Depressionen treten häufig komorbid bei somatischen Erkrankungen auf und verschlechtern hier die Prognose. Dies gilt z.B. für $25 \%$ der Patienten, die einen Herzinfarkt überlebt haben [Lavoie und Fleet, 2000]. Studien zu internetbasierten Interventionen, die auf eine Veränderung der depressiven Symptomatik bei Patienten mit koronaren Herzkrankheiten abzielen, sind bereits angelaufen [Messerli et al., 2012]. Ein anderes Beispiel ist eine niederländische Studie [van Bastelaar et al., 2011] zu einer internetbasierten Intervention gegen Depressionen bei Patienten mit Typ-1- oder Typ-2-Diabetes. Diese Intervention zeigte kleine bis mittlere Effekte auf die depressive Symptomatik.

\section{Rückfallprävention und Nachsorge}

Ein besonderes Problem bei der Behandlung von Depressionen sind die hohen Rückfallraten. In Metaanalysen wurde festgestellt, dass etwa die Hälfte der depressiven Patienten, die medikamentös behandelt wurden, und etwa ein Drittel der Patienten, die in kognitiv-verhaltenstherapeutischer Behandlung waren, innerhalb eines Jahres nach Absetzen der Antidepressiva bzw. nach Ende der Psychotherapie einen Rückfall erleiden [Williams et al., 2009; Vittengl et al, 2007]. Die Zahlen sind noch ernüchternder, wenn Patienten zum Ende der Behandlung noch an Residualsymptomen leiden [Judd et al., 1999]. Diese Zahlen unterstreichen die besondere Notwendigkeit von Nachsorgeprogrammen, die im deutschen Sprachraum vor allem von der Arbeitsgruppe um Hans Kordy (Forschungsstelle für Psychotherapie, Heidelberg) realisiert und evaluiert wurden [Kordy, 2013]. Ein Beispiel ist das Modell der Internet-Chat-Brücke, bei dem sich 6-10 Patienten im Anschluss an eine stationäre Behandlung wöchentlich für $90 \mathrm{~min}$ in einem Internet-Chatraum $\mathrm{zu}$ einer geleiteten Gruppentherapie treffen. Die Wirksamkeit und die Kosteneffektivität dieses Ansatzes wurde in verschiedenen Studien mit gemischten Patientengruppen bestätigt [Kordy et al., 2006; Bauer et al., 2011; Kordy, 2013]. Ein weiteres Beispiel einer erfolgreichen Nachsorgeintervention ist ein in Schweden entwickeltes geleitetes Selbsthilfeprogramm [Holländare et al., 2011]. Bei depressiven Patienten, die nach Abschluss einer psychotherapeutischen oder medikamentösen Behandlung nur partiell remittiert waren, führte die internetbasierte Nachsorgeintervention im Vergleich zu einer Kontrollgruppe zu einer deutlichen Reduktion der Rückfallrate (37,8\% vs. $10,5 \%$ bis 6 Monate nach Ende der traditionellen Behandlung).

\section{Fazit}

Internetbasierte psychologische Behandlungen sind angesichts der Häufigkeit von depressiven Störungen eine sinnvolle und wirksame Ergänzung zu den bestehenden Therapieangeboten. Insbesondere gestützte und ungestützte Selbsthilfeangebote im Internet sind wissenschaftlich sehr gut untersucht. Dabei steigt die Wirksamkeit bis zu einem gewissen Punkt mit der Intensität des therapeutischen Kontakts. For- 
schungsbedarf besteht besonders im Bereich der Psychotherapie über das Internet (z.B. über Skype) und im Bereich der Einbettung der internetbasierten Behandlung in die reguläre klinische Versorgung (z.B. blended treatment).

\section{Disclosure Statement}

Die Autoren erklären hiermit, dass keine Interessenskonflikte vorliegen.

\section{Literatur}

Almer S: Das Fernbehandlungsverbot als rechtliche Grenze im Einsatz Neuer Medien in der psychosozialen Versorgung. Berlin, Springer, 2008.

Andersson G, Cuijpers P: Internet-based and other computerized psychological treatments for adult depression: a meta-analysis. Cogn Behav Ther 2009;38:196-205.

Bauer S, Wolf M, Haug S, et al.: The effectiveness of internet chat groups in relapse prevention after inpatient psychotherapy. Psychother Res 2011;21: 219-226.

Berger T, Andersson G: Internetbasierte Psychotherapien: Besonderheiten und empirische Evidenz. Psychother Psych Med Psychol 2009;59:159-170.

Berger T, Caspar F: Internetbasierte Psychotherapie. Psychiatrie und Psychotherapie up2date 2011;5:2943.

- Berger T, Caspar F, Richardson R, et al.: Internetbased treatment of social phobia: a randomized controlled trial comparing unguided with two types of guided self-help. Behav Res Ther 2011b;49:158169.

-Berger T, Haemmerli K, Gubser N, et al.: Internetbased treatment of depression: a randomized controlled trial comparing guided with unguided selfhelp. Cogn Behav Ther 2011a;40:251-266.

Calear A, Christensen H: Review of internet-based prevention and treatment programs for anxiety and depression in children and adolescents. Med J Aust 2010;192:12-14.

Carlbring P, Maurin L, Torngren C, et al.: Individually-tailored internet-based treatment for anxiety disorders: a randomized controlled trial. Behav Res Ther 2011;49:18-24.

Caspar F: Technological developments and applications in clinical psychology and psychotherapy: in troduction. J Clin Psychol 2004;60:221-238.

-Christensen H, Griffiths K, Jorm A: Delivering interventions for depression by using the internet: randomised controlled trial. BMJ 2004;328:265-268.

Clarke G, Eubanks D, Reid E: Overcoming depression on the internet (ODIN): a randomized trial of self-help depression skills program with reminders. J Med Internet Res 2005;7:e14

Cuijpers P, Donker T, van Straten A, et al.: Is guided self-help as effective as face-to-face psychotherapy for depression and anxiety disorders? A meta-analysis of comparative outcome studies. Psychol Med 2010;40:1943-1957.

Cuijpers P, Donker T, Johansson R, et al.: Self-guided psychological treatment for depressive symptoms: a meta-analysis. PLoS One 2011;6:e21274.

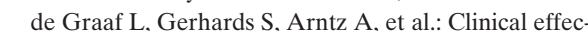
tiveness of online computerised cognitive-behavioural therapy without support for depression in primary care: randomised trial. Br J Psychiatry 2009:195:73-80.

DGPPN et al.: S3-Leitlinie / Nationale Versorgungsleitlinie Unipolare Depression-Langfassung. Berlin, Düsseldorf, DGPPN, ÄZO, AWMF. www. depression.versorgungsleitlinien.de (Zugriff am 27.06.2013)
Fournier J, DeRubeis R, Hollon S, et al.: Antidepressant drug effects and depression severity: a patientlevel meta-analysis. JAMA 2010;303:47-53.

Hedman E, Andersson G, Ljótsson B, et al.: Internetbased cognitive behavior therapy vs. cognitive behavioral group therapy for social anxiety disorder: a randomized controlled non-inferiority trial. PLoS One 2011;6:e18001.

Hedman E, Ljótsson B, Rück C, et al.: Effectiveness of internet-based cognitive behaviour therapy for panic disorder in routine psychiatric care. Acta Psychiatr Scand 2013;DOI: 10.1111/acps.12079.

Holländare F, Johnsson S, Randestad M, et al.: Randomized trial of internet-based relapse prevention for partially remitted depression. Acta Psychiat Scand 2011:124:285-294.

Hollon SD, Muñoz RF, Barlow DH, et al.: Psychosocial intervention development for the prevention and treatment of depression: promoting innovation and increasing access. Biol Psychiatry 2002;52:610630.

Johansson R, Andersson G: Internet-based psychological treatments for depression. Expert Rev Neurother 2012;12:861-870.

Johansson R, Sjöberg E, Sjöberg M, et al.: Tailored vs. standardized internet-based cognitive behavior therapy for depression and comorbid symptoms: a randomized controlled trial. PLoS One 2012;7:e36905.

Johansson R, Ekbladh S, Hebert A, et al.: Psychodynamic guided self-help for adult depression through the Internet: a randomised controlled trial. PLoS One 2012; 7:e38021.

Judd L, Paulus M, Zeller P: The role of residual subthreshold depressive symptoms in early episode relapse in unipolar major depressive disorder. Arch Gen Psychiatry 1999;56:764-765.

Kazdin AE, Blase SL: Rebooting psychotherapy research and practice to reduce the burden of mental illness. Perspect Psychol Sci 2011;6:21-37.

Kessler D, Lewis G, Kaur S, et al.: Therapist-delivered internet psychotherapy for depression in primary care: a randomized controlled trial. Lancet 2009; 374:628-634.

Kessling L, Hansen H, Demyttenaere K, et al.: Depressive and bipolar disorders: patients' attitudes and beliefs towards depression and antidepressants. Psychol Med 2005;35:1205-1213.

Kirsch I, Deacon B, Huedo-Medina T, et al.: Initial severity and antidepressant benefits: a meta-analysis of data submitted to the food and drug administration. PLoS Med 2008;5:250-268.

Knaevelsrud C, Maercker A: Internet-based treatment for PTSD reduces distress and facilitates the development of a strong therapeutic alliance: a randomized controlled clinical trial. BMC Psychiatry 2007;7:13.

Kordy H: Internet- und mediengestützte Therapie. Psychother Psych Med 2013;63:12-18.
Kordy H, Golkaramnay V, Wolf M, et al.: InternetChat-Gruppen in der Psychotherapie und Psychosomatik: Akzeptanz und Wirksamkeit einer Internet-Brücke zwischen Fachklinik und Alltag. Psychotherapeut 2006;51:144-153.

Lange A, Rietdijk D, Hudcovicova M, et al.: Interapy: a controlled randomized trial of the standardized treatment of posttraumatic stress through the internet. J Consult Clin Psychol 2003;71:901-909.

Lange A, Vermeulen H, Renckens C, et al.: De geprotocolleerde Interapy-behandeling van depressie via het internet; resultaten van een gerandomiseerde trial. Direct Ther 2005;25:27-50.

Lavoie K, Fleet R: The impact of depression on the course and outcome of coronary artery disease: review for cardiologists. Can J Cardiol 2000;16:653-662.

Leucht S, Hierl S, Kissling W, et al.: Putting the efficacy of psychiatric and general medicine medication into perspective: review of meta-analyses. Br J Psychiatry 2012;200:97-106

Lindtvedt O, Griffiths K, Sorensen K, et al.: Evaluating the effectiveness and efficacy of unguided inter net-based self-help intervention for the prevention of depression: a randomized controlled trial. Clin Psychol Psychother 2013;20:10-27.

Ly KH, Carlbring P, Andersson G: Behavioural activation-based guided self-help treatment administered through a smartphone application: study protocol for a randomized controlled trial. Trials 2012 13:62.

Merry S, Stasiak K, Shepherd M, et al.: The effectiveness of SPARX, a computerised self-help intervention for adolescents seeking help for depression: randomised controlled non-inferiority trial. BMJ 2012;344:e2598.

Messerli N, Barth J, Berger T: The InterHerz project - a web-based psychological treatment for cardiac patients with depression: study protocol of a randomized controlled trial. Trials 2012;13:245.

Meyer B, Berger T, Caspar F, et al.: Effectiveness of a novel integrative online treatment for depression (deprexis): randomized controlled trial. J Med Internet Res 2009;11:e15.

Mohr D, Ho J, Duffecy J, et al.: Perceived barriers to psychological treatments and their relationship to depression. J Clin Psychol 2010;66:394-409.

Mohr D, Ho J, Duffecy J, et al.: Effect of telephoneadministered vs face-to-face cognitive behavioral therapy on adherence to therapy and depression outcomes among primary care patients: a randomized trial. JAMA 2012;307:2278-2285.

Morgan A, Jorm A, Mackinnon AJ: Email-based promotion of self-help for subthreshold depression: Mood Memos randomised controlled trial. $\mathrm{Br} \mathrm{J}$ Psychiatry 2012;200:412-418.

Moritz S, Schröder J, Meyer B, et al: The more it is needed, the less it is wanted: attitudes toward faceto-face intervention among depressed patients undergoing online treatment. Depress Anxiety 2013; 30;157-167. 
Muñoz RF: Using evidence-based internet interventions to reduce health disparities worldwide. J Med Internet Res 2010;12:e60.

- Preschl B, Maercker A, Wagner B: The working alliance in a randomized controlled trial comparing online with face-to-face cognitive-behavioral therapy for depression. BMC Psychiatry 2011;11:189.

Preschl B, Wagner B, Forstmeier S, et al.: E-health interventions for depression, anxiety disorders, dementia and other disorders in older adults: a review. J Cyberther Rehabil 2012;4:371-385.

Raue P, Schulberg H, Heo M, et al.: Patients' depression treatment preferences and initiation, adherence, and outcome: a randomized primary care study. Psychiatr Serv 2009;60:337-343.

Ruwaard J, Schrieken B, Schrijver M, et al.: Standardized web-based cognitive behavioural therapy of mild to moderate depression: a randomized controlled trial with a long-term follow-up. Cogn Behav Ther 2009;16:1-16.

Ruwaard J, Lange A, Schrieken B, et al.: The effectiveness of online cognitive behavioral treatment in routine clinical practice. PLoS One 2012;7:440089.
Spek V, Cuijpers P, Nyklicek I, et al.: Internet-based cognitive-behaviour therapy for symptoms of depression and anxiety: a meta-analysis. Psychol Med 2007:37:319-328.

Titov N: Internet-delivered psychotherapy for depression in adults. Curr Opin Psychiatry 2011;24:18-23.

Titov N, Dear BF, Schwencke G, et al.: Transdiagnostic internet treatment for anxiety and depression: a randomised controlled trial. Behav Res Ther 2011; 49:411-452.

van Bastelaar K, Cuijpers P, Pouwer F, et al.: Development and reach of a web-based cognitive behavioural therapy programme to reduce symptoms of depression and diabetes-specific distress. Patient Educ Couns 2011;84:49-55.

van Voorhees B, Fogel J, Reinecke M, et al.: Randomized clinical trial of an Internet-based depression prevention program for adolescents (Project CATCH-IT) in primary care: 12-week outcomes. J Dev Behav Pediatr 2009;30:23-37.

Vernmark K, Lenndin J, Bjärehed J, et al.: Internet administered guided self-help versus individualized e-mail therapy: a randomized trial of two versions of CBT for major depression. Behav Res Ther 2010;48:368-376.
Vittengl J, Clark L, Dunn T, et al.: Reducing relapse and recurrence in unipolar depression: a comparative meta-analysis of cognitive-behavioral therapy's effects. J Consult Clin Psychol 2007:75:475-488.

Wagner B, Lange A: Internetbasierte Psychotherapie «Interapy»; in Bauer S, Kordy H (eds): E-MentalHealth. Berlin, Springer, 2008, pp 105-120.

Walther J: Computer-mediated communication: impersonal, interpersonal, and hyperpersonal interaction. Commun Res 1996;23:3.

Williams N, Simpson A, Simpson K, et al.: Relapse rates with long-term antidepressant drug therapy: a meta-analysis. Hum Psychopharmacol 2009;24: 401-408.

Wittchen H, Holsboer F, Jacobi F: Met and unmet needs in the management of depressive disorders in the community and primary care: The size and the breadth of the problem. J Clin Psychiatry 2001; 62:993-1000.

Wittchen H, Jacobi F, Rehm J: The size and burden of mental disorders and other disorders of the brain in Europe 2010. Eur Neuropsychopharmacol 2011; 21:655-679. 